\title{
A REMARK CONCERNING THE NECESSARY CONDITION OF WEIERSTRASS*
}

\author{
BY E. J. MCSHANE $\dagger$
}

Let us consider a class $\Omega$ of rectifiable curves $C$ lying in a point set $A$ of $n$-dimensional space, and an integral $F(C)=\int_{c} f\left(x, x^{\prime}\right) d s$, where $x=\left(x^{1}, \cdots, x^{n}\right)$ and $s$ connotes that we use the length of arc as parameter. Suppose that a certain curve $C: x=x(s)$ minimizes $F(C)$ in $\Omega$, and denote by $L$ the set of points of $C$ which are interior to $A$ and of indifference with respect to $\Omega$ and $A$. Then for almost all points of $L$ we have $E\left(x(s), x^{\prime}(s), \bar{x}^{\prime}\right) \geqq 0$ for all sets of numbers $\bar{x}^{\prime}$. Given now a particular point $x\left(s_{0}\right)$ of $L$; when can we say that the inequality holds at $x\left(s_{0}\right)$ ?

It has already been shown $\S$ that the inequality holds if $x^{\prime}\left(s_{0}\right)$ exists, $\Sigma\left[x^{i^{\prime}}\left(s_{0}\right)\right]^{2}>0$, and the $x^{i^{\prime}}(s)$ are all approximately continuous at $s_{0}$. We will now show that the inequality also holds if $\Sigma\left(x^{i^{\prime}}\left(s_{0}\right)\right)^{2}=1$. (As is well known, this sum never exceeds 1 , and is equal to 1 almost everywhere.)

Suppose then that $\Sigma\left[x^{i^{\prime}}\left(s_{0}\right)\right]^{2}=1$ and that in contradiction to our statement there exists an $\bar{x}^{\prime}$ such that $E\left(x\left(s_{0}\right), x^{\prime}\left(s_{0}\right), \bar{x}^{\prime}\right)$ $=-2 k<0$. Denote by $\alpha(s)$ the angle between $x^{\prime}(s)$ and $x^{\prime}\left(s_{0}\right)$. The function

$$
\begin{aligned}
\phi(s) & =\frac{d}{d s}\left[\sum x^{i}(s) x^{i^{\prime}}\left(s_{0}\right)\right]=\sum x^{i^{\prime}}(s) x^{i^{\prime}}\left(s_{0}\right) \\
& =\left\{\sum\left[x^{i^{\prime}}(s)\right]^{2}\right\}^{1 / 2}\left\{\sum\left[x^{i^{\prime}}\left(s_{0}\right)\right]^{2}\right\}^{1 / 2} \cos \alpha(s)
\end{aligned}
$$

is defined for almost all values of $s$, and $|\phi(s)| \leqq|\cos \alpha(s)|$. By the continuity of $E$, we can find positive numbers $\epsilon, \delta$ such that $E\left(x(s), x^{\prime}(s), \bar{x}^{\prime}\right)<-k$ for all $s$ such that $\left|s-s_{0}\right| \leqq \epsilon, \phi(s) \geqq 1-\delta$; and if $\epsilon$ be small enough, $x(s)$ will be in $L$. But $\phi\left(s_{0}\right)=1$ and $\phi(s)$

* Presented to the Society, April 3, 1931.

$\dagger$ National Research Fellow.

$\ddagger$ L. Tonelli, Fondamenti di Calcolo delle Variazioni, vol. 2, p. 87. E. J. McShane, On the necessary condition of Weierstrass, etc., Annals of Mathematics, vol. 32.

§ E. J. McShane, loc. cit. 
is a derivative; therefore* there exists on $\left[s_{0}-\epsilon, s_{0}+\epsilon\right]$ a set of positive measure for which $\phi(s)>1-\delta$, ". ..., which contradicts the theorem quoted above."

Ohio State University

\title{
A CORRECTION AND AN ADDITION
}

\author{
BY G. E. RAYNOR
}

1. A Correction. In a former paper $\dagger$ by the author the minus sign on the right side of equation (4), page 888 , makes the notations of equations (4) and (5) for the function $G$ inconsistent. This difficulty may be removed by changing the sign of $G$ throughout the paper wherever the first argument of $G$ has $r_{1}$ in the denominator. This change makes the first footnote on page 888 superfluous and it should be deleted. The second argument of $G$ in equations (9) and (20) should be 0 instead of $\theta$.

2. An Addition. The mean value of the function $\Phi$ over the circle $C_{2}$ was considered, in the paper, for the case of the singular point $P$ outside of $C_{2}$ and for the case of $P$ inside of $C_{2}$. The question naturally arises as to what the situation is in case $P$ lies on $C_{2}$. This third case is not, however, of much interest since the integral

$$
\int_{C_{2}} \Phi d s
$$

which is now in general improper, will not in general exist. This may readily be verified for the function

$$
\Phi=\left(\frac{r^{2}}{r_{1}^{2}}-\frac{r_{1}^{2}}{r^{2}}\right) \cos 2 \theta
$$

integrated over the circle $C_{2}$, whose equation is $\rho=r_{1} \sin \theta$. It will be found that even the principal value of the above integral is infinite while of course the value of $\Phi$ at the center of $C_{2}$ is finite.

LEHIGH UNIVERSITY

* Hobson, Theory of Functions of a Real Variable, vol. 1, \$403.

$\dagger$ On the extension of the Gauss mean-value theorem to circles in the neighborhood of isolated singular points of harmonic functions, this Bulletin, vol. 36 (1930), pp. 887-893. 